\title{
WHAT DO WE REALLY KNOW ABOUT CLOUD FORMATION?
}

\author{
BRUCE G. ELMEGREEN \\ IBM Research Division, T.J. Watson Research Center \\ P.O. Box 218, Yorktown Heights, NY 10598, USA \\ bge@watson.ibm.com
}

\begin{abstract}
Four processes of molecular cloud formation are discussed: formation in swept-up shells, coagulation of smaller clouds, condensation in larger clouds, and compression in a supersonically turbulent medium. Examples and constraints for each process are given.
\end{abstract}

\section{Introduction}

Most cloud formation mechanisms that have been proposed can be placed into one or more of the following four categories: (1) cloud formation by the collection of gas into a shell or ring in the ambient interstellar medium, followed by the gravitational collapse of this gas along the periphery of the shell or ring into new self-gravitating clouds; (2) cloud formation by the coagulation of smaller clouds; (3) cloud formation by condensation from larger clouds, and (4) cloud formation by compression between converging eddies in a supersonically turbulent medium. Here we review each mechanism and consider the conditions that are necessary to make a self-gravitating cloud in which stars form.

\section{Cloud Formation by Collection and Collapse in Shells and Rings}

There are many examples of hollow round holes, shells, or rings in the interstellar medium of our Galaxy (Heiles 1979), M31 (Brinks and Bajaja 1986), M33 (Deul and Hartog 1990), HoII (Puche et al. 1992), and a few other galaxies (e.g., Irwin 1990). These holes were presumably made by expansion away from central pressure sources that could be $O B$ associations (Bruhweiler et al. 1980), stray supernovae, high velocity cloud impacts 
(Tenorio-Tagle 1981), and other energetic events. A review of interstellar holes and shells is in Tenorio-Tagle and Bodenheimer (1988). An extensive review of star formation in shells and rings is in Elmegreen (1992).

Small holes and shells with diameters between $1 \mathrm{pc}$ and $50 \mathrm{pc}$ often occur in $\mathrm{OB}$ associations. Occasionally there is active star formation along the periphery of these cavities (Elmegreen and Lada 1977; Thronson et al. 1985; Junkes et al. 1992; Laval et al. 1992). This star formation was presumably triggered by the expansion that made the cavity because the embedded stars along the periphery are usually much younger than the source of the high pressure, and they are sometimes moving away from the expansion center along with the rest of the gas (Thronson et al. 1985). The clouds in which these young stars form is probably residual gas from the cloud that originally made the older $\mathrm{OB}$ association. In that case, the triggering process is related to the rearrangement of the old cloud, not the formation of a new cloud.

Many large shells and holes are visible in HI maps of the Large Magellanic Clouds. Westerlund and Mathewson (1966), Dopita et al. (1985), Goudis and Meaburn (1978), Wang and Helfand (1991), Bruhweiler et al. (1991) and others have studied these regions. Many of these also show recent star formation along their peripheries. Sometimes these regions are again just examples of cloud rearrangement, or of the formation of new dense cores inside existing clouds, but sometimes they are regions of new cloud formation.

It is often difficult to tell the difference between cloud rearrangement and cloud formation because both can have a morphology where young stars are located on the periphery of an old expanding shell. The difference between these two cases is important however. In the first case, the process is involved with cloud destruction and the eventual cessation of star formation. The important point here is that star formation does not stop the first time a cloud is broken apart because many dense, self-gravitating fragments remain. It may take three or more generations of star formation to completely disassemble a cloud. All of the time these new stars form, the total mass of dense gas in the region can steadily decrease. In the second case, the process of expansion and cavity formation is involved with new cloud formation. Then the total mass of dense star-forming material either increases with time or remains approximately constant as old disrupted clouds are replaced by new star-forming clouds.

There are two ways in which an expanding shell can make new dense clouds, one in which the pressure from the expansion squeezes existing diffuse clouds into dense self-gravitating clouds, and another in which the accumulated material at the edge of the expansion collapses gravitationally into new clouds separated along the periphery. The first case of cloud 
squeezing has been studied by Klein et al. (1985) and others. The second case of peripheral collapse has been studied by McCray and Kafatos (1987), Comeron and Torra (1994), and Elmegreen (1994b).

The time scales for expanding shells and rings to collapse along their periphery and make new self-gravitating clouds are given by the equations (Elmegreen 1994b):

$$
t_{\text {shell }}=\frac{1.25}{\left(G \rho_{0} \mathcal{M}\right)^{1 / 2}} ; t_{\text {ring }}=\frac{1.5}{\left(G \rho_{0}\right)^{1 / 2} \mathcal{M}}
$$

where $\mathcal{M}=V / c$ for expansion speed $V$ and $\mathrm{rms}$ speed inside the shell $c$. The density external to the shell is $\rho_{0}$. These equations assume the expansion proceeds as in a wind-blown bubble, as $R \propto t^{0.6}$. The resulting collapse times are rather long for the ambient medium in a typical galaxy disk. They are equal to approximately $10^{8}$ years divided by $\mathcal{M}^{1 / 2}$ and $\mathcal{M}$ for shells and rings, respectively, if the density is around 1 atom $\mathrm{cm}^{-3}$. For small $\mathcal{M}$, this is a longer time than the duration of the pressure that drives the expansion, so we obtain our first constraint for this mechanism of cloud formation:

CONSTRAINT 1: The expansion has to be powerful enough and longlasting enough to make $\mathcal{M}$ large for a sufficiently long time that the collapse can occur before the shell erodes and disperses.

This means that the peripheral gas will collapse into new self-gravitating clouds if the expansion speed $V$ is fairly large, or the internal dispersion in the swept-up gas, $c$, is small, or the ambient density, $\rho_{0}$, is high, or the duration of the compression is long. Thus, collapses like this are fairly common in existing molecular clouds because $\rho_{0}$ is large, making $\left(G \rho_{0}\right)^{-1 / 2}$ comparable to the lifetime of an O-type star that forms in the cloud. At low densities, however, or for very short-lived pressures, many expansions should just disperse the gas back into the ISM without any significant gravitational collapse, except possibly for very late times, even after the erosion begins, when $c$ may drop to a low value. Then numerous tiny clouds or globules can form in the old shell.

A second condition for collapse is that the collapse time cannot be too much larger than the time for the shell or ring to shear away because of Coriolis and tidal forces in the background galaxy. If Coriolis forces are large because of a rapid galaxy rotation rate compared to $\left(G \rho_{0}\right)^{-1 / 2}$, then the expansion will just swirl around or close back up (Palous et al. $1990)$. This constraint can be written in terms of the $Q$ parameter $Q=$ $\kappa c_{0} /\left(\pi G \sigma_{0}\right)$ for epicyclic frequency $\kappa$, ambient velocity dispersion $c_{0}$, and mass column density in the disk $\sigma_{0}$ (Elmegreen 1994b). This leads to our second constraint: 
CONSTRAINT 2: Collapse must occur before shear, requiring that

$$
Q<0.66 \mathcal{M} \text {. }
$$

This second constraint implies that molecular cloud formation is likely to occur by this mechanism in regions of galaxies where $Q$ is low, which is where spiral structure and gravitational instabilities form molecular clouds by a competitive mechanism (Section 4). It also suggests that because $\kappa$ is very high in the inner regions of galaxies, scaling approximately as $1 / r, \sigma$ has to be very high as well. This goes a long way towards accounting for the starburst phenomenon regardless of the mechanism of star formation in starburst galaxies (Elmegreen 1994a).

Another implication of constraint 1 , which also applies to starburst nuclei, is that in regions where the ambient density is very high, exceeding $\sim 10^{3} \mathrm{~cm}^{-3}$, the time scale for triggering is shorter than the lifetime of an O-type star for the whole interstellar medium (and not just for the cloud in which the $\mathrm{O}$-star formed, as mentioned above). This implies that $\mathcal{M}$ should always be fairly large at the time of collapse, and the time scale could be significantly shorter than $\left(G \rho_{0}\right)^{-1 / 2}$. Then the formation of molecular clouds in shells and rings would be more important than their formation in ambient instabilities, which operate on the time scale $\left(G \rho_{0}\right)^{1 / 2}$.

As a result of these considerations, we make the following two predictions:

PREDICTION 1: GMC formation in giant shells should occur in about the same radial range in a galaxy disk as GMC formation in spiral arms and in giant condensations having the ambient Jeans mass (i.e., low $Q$ ).

PREDICTION 2: Starburst nuclei, where the gas density is very large, should have a high fraction of their star formation triggered in irregular shells and other swept-up debris, rather than in clouds with the ambient Jeans mass (i.e., $t_{\text {shell }}<t_{O B}$ star $)$.

A similar mechanism for cloud and star formation is related to the formation of shells or compressed gas structures during the collision between a high velocity cloud and the galactic plane. A recent model for this process is in Lepine and Duvert (1994), who apply it to the Orion OB association and other regions that are far from the midplane. These authors point out that an important implication of this model is that the old stars will lie ahead of the gas and young stars in the track of the colliding cloud because these old stars, having formed first, did not decelerate along with the rest of the gas after they formed in the compressed layer. A second point is that the midplane below the high latitude clouds that form this way should be relatively free of other clouds because of the clearing effect of the high velocity impact. Third, the leading, oldest stars should be relatively free of gas because the gas in which they formed was pushed back by the ram 
pressure of the galactic plane at the time they were exposed. These aspects of the model appear to be true for several regions around the Sun.

\section{Cloud Formation by Coagulation of Smaller Clouds}

In the Oort (1954) model of cloud formation, small clouds randomly hit each other and stick together until they become large enough to collapse gravitationally. Then star formation breaks them apart and the coagulation process starts over again. This may work for diffuse cloud collisions, which Oort considered, because such collisions can be softened by magnetic fields, and because star formation does not occur in diffuse clouds until they become significantly self-gravitating. It may not work for GMC collisions, because small GMCs should form stars and disrupt before they collide with each other, and because small GMCs probably cannot stick together if they move relative to each other at the full rms speed of the interstellar medium.

Sticking collisions for equal mass clouds require a sufficiently low velocity that the collision fragments are gravitationally bound to each other after the collision is over. This implies

$$
v_{\text {rel }} \leq 2.5 v_{\text {esc }}
$$

for relative collision speed $v_{r e l}$ and cloud escape speed $v_{e s c}$ (Pumphrey and Scalo 1983). Using the size-linewidth relation for molecular clouds (Solomon et al. 1987) and the assumption that the clouds are virialized allows us to convert this relation into a mass limit for sticking, non-magnetic collisions:

$$
M>4.3 \times 10^{4}\left(\frac{v_{r m s}}{5 \mathrm{kms}^{-1}}\right)^{4} \mathrm{M}_{\odot}=1.6 \times 10^{6}\left(\frac{v_{S D W}}{30 \mathrm{kms}^{-1}}\right)^{4} \mathrm{M}_{\odot} .
$$

The first of these expressions is useful for random collisions of clouds moving at the relative speed of $6^{1 / 2}$ times the observed one-dimensional rms speed, and the second is useful for collisions at spiral density wave shocks, where the relative speed is large. Evidently small GMCs (e.g., $10^{3} \mathrm{M}_{\odot}$ ) in the Oort model are not likely to stick together if they collide with each other at the full $\mathrm{rms}$ speed of the interstellar medium.

Coagulation models are still reasonable, however, but they require some modifications from the original Oort picture. Most GMCs could accrete smaller clouds or diffuse clouds, which would dissipate so much energy during the collision that the velocity threshold may not apply. GMCs could also accrete other GMCs that are co-moving in space, i.e., not moving relative to each other at the full $\mathrm{rms}$ speed that is observed. This situation is likely to apply to turbulent models of the ISM because the relative speed between parcels of gas is small on small scales. GMCs that form in an expanding shell may coalesce too because they are co-moving. 
Another possibility is that clouds collide and ultimately stick together - even at high velocities - because the cloud filling factor is nearly unity. This situation may arise in spiral arms or at the ends of the bars in barred galaxies where ISM streamlines converge and the clouds crowd together (Kenney and Lord 1991).

In addition, diffuse clouds could stick together magnetically to make a GMC, as mentioned above. In this case, the clouds would not have to touch each other physically to stick together, they need only have their magnetic field lines link up and get tangled. The cross section for such tangling is relatively large (Clifford and Elmegreen 1983) so such collisions should be frequent or even continuous.

Another aspect of cloud collisions is that even if clouds do not stick together, the collisions between them help decrease the turbulent speed in the ISM and this leads to GMC formation by macroscopic thermal instabilities. This instability is one in which a local region with, say, an excess in density, has an excess rate of cloud collisions and an excess rate of dissipation of random cloud motions. Because the average density does not change during this dissipation process, the result is a slight decrease in the turbulent pressure. The surrounding clouds continuously hit this region, however, and these impacts transfer inward-directed momentum to the cloud fragments from the collisions. This acts like an external pressure on the cooling region, which then begins to cave-in, leading to a higher density and even more cloud collisions. Eventually the region has a core with a low velocity dispersion and a high density, and it has a continuing accretion of the ambient high dispersion clouds. The core then grows arbitrarily large. Such a mechanism of cloud formation has been studied by Struck-Marcell and Scalo (1984), Tomisaka (1987), and Elmegreen (1989). It may be present in most $\mathrm{N}$-body simulations with particles for clouds. When gravity is included, the instability becomes a very powerful driver for giant cloud formation.

These considerations lead to the following predictions about diffuse and molecular clouds:

PREDICTION 1: Diffuse clouds that form by the macroscopic thermal instability should appear in quiescent regions (not shells or shock fronts) with low internal rms speeds and high internal densities in approximate turbulent pressure equilibrium with the surrounding diffuse cloud population. Molecular cores and even star formation may appear in their densest self-gravitating parts.

PREDICTION 2: Orbit crowding in galactic spiral arms and near the ends of the bars in barred galaxies should make the diffuse cloud filling factor near unity, which implies ISM compression factors of around 20. This drives the coagulation of clouds into dust lanes or giant complexes, and this is a precursor to GMCs and star formation. 


\section{Cloud Formation by Condensation from Larger Clouds}

The largest cloud complexes in spiral arms typically have masses of $\sim 10^{7}$ $\mathrm{M}_{\odot}$ (e.g., Knapen et al. 1993; Garcia-Burillo et al. 1993), which is larger than the mass of the largest GMCs in our Galaxy. Most spiral arm GMCs are in the cores of these complexes (e.g., Grabelski et al. 1987), so they probably formed by condensation from the larger clouds (Elmegreen and Elmegreen 1983).

The $10^{7} \mathrm{M}_{\odot}$ "superclouds" have a mass comparable to the Jeans' mass at the average gas density and velocity dispersion in the Galaxy. The Jeans' length and mass for disk collapse are

$$
\lambda=\frac{2 \hat{c}^{2}}{G \sigma} \approx 2.5 \mathrm{kpc} \quad ; \quad M=\frac{\hat{c}^{4}}{G^{2} \sigma} \approx 10^{7} \mathrm{M}_{\odot}
$$

where $\hat{c}^{2}=\gamma_{e f f} c^{2}+v_{A}^{2}$ is an effective mean squared velocity dispersion including dissipation and magnetic fields. Here, $c$ is the rms dispersion in 1-dimension, $\gamma_{e f f}$ is an effective ratio of specific heats from the energy equation, and $v_{A}$ is the Alfven speed for the component of the magnetic field perpendicular to the collapse. A more complete derivation of these quantities, including an expression for $\gamma_{e f f}$, is in Elmegreen $(1991,1994 \mathrm{c})$.

Large cloud complexes like these are probably the result of gravitational instabilities in the spiral arms. Conditions for a spiral arm to be unstable were discussed by Elmegreen $(1979,1994 \mathrm{c})$, Balbus and Cowie (1985), and Balbus (1988). The conditions can be most simply written in terms of the dimensionless parameters $G \mu / \hat{c}^{2}$ and $\rho_{\text {arm }} / \rho_{\text {crit,ave }}$, both of which must exceed 1 , the first so that the arm is strongly self-gravitating, and the second so that the collapse time is less than the flow-through time. Here $\mu$ is the mass/length along the arm, $\rho_{a r m}$ is the gas density in the arm and $\rho_{\text {crit,ave }}$ is the average critical density at that radius in the galaxy, given by $\rho_{\text {crit }, \text { ave }}=\rho_{\text {ave }} Q$ for instability parameter $Q=\kappa \hat{c} /(\pi G \sigma)$ with epicyclic frequency $\kappa$ and mass/area $\sigma$ in the plane.

Spiral arms trigger this instability because the gas is dense (self-gravity is strong), the magnetic field is strong (the angular momentum of the gas is readily removed during the collapse), the rate of shear is low (the collapse proceeds in a parallel fashion along the arm, producing "pearls on a necklace" of clouds rather than spirals within spirals), and tidal forces are low (Jean-mass clouds, which necessarily have low densities after virialization because of their large mass, are not prevented from forming by Galactic tidal forces).

In a galaxy with strong spiral arms, most of the star formation occurs in the giant cloud complexes that occupy these arms. There is apparently relatively little star formation in shells or smaller isolated clouds. This 
makes one suspect that the dominant mode of star formation varies from galaxy to galaxy, depending on the presence or lack of spiral waves. This observation should not be interpreted to imply that the star formation rate per unit gas mass varies in a similar way. In fact, this quantity is surprisingly constant, to within a factor of $\sim 5$ (e.g., Kennicutt 1983; Wiklind and Henkel 1989; Thronson, et al. 1989; Devereux and Young 1991, Sage 1993). Instead it is the mode or mechanism of star formation that varies: shells and triggered star formation in spiral-less galaxies like HoII (Puche et al. 1992) replace condensation-type star formation in $10^{7} \mathrm{M}_{\odot}$ clouds in spiral galaxies like NGC 4321. Apparently the high density, low shear and low tidal forces in spiral arms affords them a condition in which supercloud formation dominates all other modes of star formation. When there are no spirals, such clouds either fail to form, or form with difficulty and a slow rate, thus permitting other forms of star formation to dominate.

\section{Cloud Formation by Compression in Supersonic Turbulence}

The last mechanism of cloud formation considered here has received relatively little attention compared to the others, but seems now, with our generally increased attention to interstellar turbulence, to be at least as important as the others. This mechanism has clouds form randomly, spontaneously, and continuously in a supersonically turbulent medium as high speed eddies crash and compress themselves, dissipating kinetic energy and producing high densities. The intercloud medium in this model is fastmoving: it exerts a ram pressure on the surfaces of the intervening clouds that is comparable to the isotropic turbulent pressure inside the clouds.

The most obvious application of this model is to the formation of clumps inside clouds (Sasao 1973; Scalo 1990; Elmegreen 1990; Falgarone and Phillips 1990; Porter, et al. 1992; Yue et al. 1993). Such a formation mechanism presumably accounts for the fractal appearance of the cloud boundaries and the hierarchy of scales inside the clouds. Unfortunately, neither of these properties of a GMC have been demonstrated theoretically with turbulence simulations nor have they been reproduced in a laboratory. Thus the interpretation of GMC clumps in terms of structures made by supersonic turbulence is somewhat speculative at the moment, but it is extremely compelling and natural, making the arguments highly persuasive.

The point of view taken in this section is to propose that not only are the clumps inside of clouds likely to have formed by compression in supersonic turbulence, but also the whole clouds themselves. That is, whole GMCs form by compression in supersonic turbulence on extremely large scales, up to a galactic scale height or more. The gas becomes self-gravitating during the compression, as in the cloud-clump model by Elmegreen (1993), 
and then GMCs form by collapse inside the turbulence-compressed layers. Diffuse clouds presumably form the same way but in smaller eddies, at slower speeds, and without enough mass to be strongly self-gravitating.

The advantage of this turbulent model is that it can account for the formation of interstellar structures on all scales that are not connected directly with either shells or superclouds from spiral arm instabilities. Turbulence can account for the IRAS cirrus clouds and high latitude clouds, molecular or atomic. It may also account for the random positions of GMCs inside superclouds, as shown, for example, by the maps in Grabelski et al. (1987). It might even account for the random positions of clouds and star formation in flocculent galaxies, which have no spiral waves.

The turbulent model makes the following predictions:

PREDICTION 1: GMC positions inside a supercloud should have the same geometric character as clump positions inside a GMC.

PREDICTION 2: Young GMCs without obvious connections to shells and direct sources of high pressure should have surrounding intercloud flows that are converging on the cloud.

PREDICTION 3: GMCs and diffuse clouds should be interconnected and part of the same turbulent structures, with GMCs representing only the self-shielded and self-gravitating regions of the larger scale complexes.

PREDICTION 4: Star formation in turbulence-compressed GMCs should have an intermittent quality in time, and a fractal quality in space.

Perhaps with these predictions, observers can find evidence for clouds that have formed this way.

\section{References}

Balbus, S.A. (1988) ApJ, 324, 60.

Balbus, S.A. and Cowie, L.L. (1985) $A p J, 297,61$.

Brinks, E. and Bajaja, E. (1986) $A \cup A, 169,14$.

Bruhweiler, F.C., Gull, T.R., Kafatos, M. and Sofia, S. (1980) ApJ, 238, L27.

Bruhweiler, F.C., Fitzurka, M.A. and Gull, T.R. (1991) ApJ, 370, 551

Clifford, P. and Elmegreen, B.G. (1983) MNRAS, 202, 629.

Comeron, F. and Torra, J. (1994) $A p J, 423,652$.

Deul, E.L. and Hartog, R.H. (1990) $A \mathcal{E} A, 229,362$.

Devereux, N.A. and Young, J.S. (1991) $A p J, 371,515$.

Dopita, M.A., Mathewson, D.J. and Ford, W.L. (1985) ApJ, 297, 599

Elmegreen, B.G. (1979) ApJ, 231, 372.

Elmegreen, B.G. (1989) $A p J, 344,306$.

Elmegreen, B.G. (1990) $A p J, 361$, L77.

Elmegreen, B.G. (1991) ApJ, 378, 139.

Elmegreen, B.G. (1992) in Star Formation in Stellar Systems, eds. G. Tenorio-Tagle, M.

Prieto and F. Sanchez, Cambridge, Cambridge University Press, 381.

Elmegreen, B.G. (1993) ApJ, 419, L29.

Elmegreen, B.G. (1994a) ApJ, 425, L73.

Elmegreen, B.G. (1994b) $A p J, 427,384$.

Elmegreen, B.G. (1994c) ApJ, 433, 39. 
Elmegreen, B.G. and Lada, C.J. (1977) ApJ, 214, 725.

Elmegreen, B.G. and Elmegreen, D.M. (1983) MNRAS, 203, 31.

Falgarone, E. and Phillips, T. (1990) ApJ, 359, 344.

Garcia-Burillo, S., Guelin, M. and Chernicharo, J. (1993) $A \mathscr{E A}, 274,123$.

Goudis, C. and Meaburn, J. (1978) $A \mathscr{E A}, 68,189$.

Grabelsky, D.A., Cohen, R.S., May, J., Bronfman, L. and Thaddeus, P. (1987) $A p J, 315$, 122.

Heiles, C. (1979) $A p J, 229,533$.

Irwin, J.A. and Seaquist, E.R. (1990) ApJ, 353, 469.

Junkes, N., Fürst, E. and Reich, W. (1992) $A \mathscr{E} A$, 261, 289.

Kenney, J.D.P. and Lord, S.D. (1991) $A p J, 381,118$.

Kennicutt, R.C. (1983) ApJ, 272, 54.

Klein, R.I., Whitaker, R.W. and Sandford, M.T., II (1985) in Protostars and Planets II, edS. D.C Black and M. S. Matthews, Univ. Arizona, Tucson, 33.

Knapen, J.H., Cepa, J., Beckman, J.E., Soledad Del Rio, M. and Pedlar, A. (1993) ApJ, 416, 563.

Laval, A., Rosado, M., Bodesteix, J., Georgelin, Y.P., Le Coarer, E., Marcelin M. and Viale, A. (1992) $A \& A, 253,213$.

Lepine, J.R.D. and Duvert, G. (1994) $A \mathscr{B} A, 286,60$.

McCray, R. and Kafatos, M. (1987) $A p J, 317,190$.

Oort, J.H. (1954) Bull.Astr.Inst.Neth, 12, 177.

Palouš, J., Franco J. and Tenorio-Tagle, G. (1990) $A \& A, 227,175$.

Porter, D.H., Pouquet, A. and Woodward, P.R. (1992) Theoret.Comp Fluid Dyn., 4, 13.

Pumphrey, W.A. and Scalo, J.M. (1983) $A p J, 269,531$.

Puche, D., Westpfahl, D. and Brinks, E. (1992) $A J, 103,1841$.

Sage, L.J. (1993) $A \mathscr{E} A, 272,123$.

Sasao, T. (1973) PASJ, 25, 1.

Scalo, J. (1990) in Physical Processes in Fragmentation and Star Formation, eds. R. Capuzzo.Dolcetta, C. Chiosi and A. Di Fazio Kluwer, Dordrecht, 151.

Solomon, P.M., Rivolo, A.R., Barrett, J. and Yahil, A. (1987) ApJ, 319, 730.

Struck-Marcell, C. and Scalo, J.M. (1984) $A p J, 277,132$.

Tenorio-Tagle, G. (1981) $A \& A, 94,338$.

Tenorio-Tagle, G. and Bodenheimer, P. (1988) ARAA, 26, 146.

Tomisaka, K. (1987) PASJ, 39, 109.

Thronson, H.A., Lada, C.J. and Hewagama, T. (1985) ApJ, 297, 662.

Thronson, H.A., Tacconi, L., Kenney, J., Greenhouse, M.A., Margulis, M., TacconiGarman, L. and Young, J. (1989) ApJ, 344, 747.

Wang, Q. and Helfand, D. (1991) ApJ, 379, 327.

Westerlund, B.E. and Mathewson, D.S. (1966) MNRAS, 131, 371.

Wiklind, T. and Henkel, C. (1989) $A \mathscr{B} A, 225,1$.

Yue, Z.Y., Zhang, B., Winnewisser, G. and Stutzki, J. (1993) Ann.Physiks, 2, 9. 